Pacific

Journal of

Mathematics

TWISTED LINKING NUMBERS VIA REPRESENTATIONS OF FUNDAMENTAL GROUPS

Kenichi KaWAGOE 


\title{
TWISTED LINKING NUMBERS VIA REPRESENTATIONS OF FUNDAMENTAL GROUPS
}

\author{
Kenichi KaWAGOE \\ Dedicated to Professor Mitsuyoshi Kato for his 60th Birthday
}

\begin{abstract}
Using representations of fundamental groups, we introduce the concept of twisted linking numbers. If the corresponding representation is trivial, the twisted linking number coincides with the linking number. In the case of a nontrivial representation, however, this is not necessarily true, which implies that the twisted linking number can detect the nontriviality of an embedding of $\mathbb{S}^{1}$. An example is given for which the linking number is trivial but the twisted linking number is not.
\end{abstract}

\section{Introduction.}

A linking number is an invariant of an oriented 2-component link. Linking numbers are frequently used to study Alexander polynomials of knots and links, and coverings of certain spaces such as the complement of a knot or the complement of a link. There are several definitions of linking numbers, corresponding to the various contexts within which they are studied: The theories regarding fundamental groups, the Gauss integral, coverings of complements of knots, diagrams, etc. For example, there are eight equivalent definitions in [7]. In the context of knot complements, linking number is defined in terms of the fundamental group of the knot complement and its abelianization. Since the abelianization gives only a 1-dimensional representation, the following is a natural question: If we consider nontrivial representations instead of the 1-dimensional abelian representation of the abelianization, how do we define the extension of a linking number? If it can be defined, what does this extended concept represent?

The purpose of this paper is to introduce the notion of linking numbers through representations of fundamental groups. For the sake of generality, we consider links with $r$ components $(r \geq 1)$. Let $L$ be an oriented, nonsplit link in $\mathbb{S}^{3}$. We use the Wirtinger presentation as the presentation of $\pi L=\pi_{1}\left(\mathbb{S}^{3} \backslash L\right)$. Let $K$ be an embedding of $\mathbb{S}^{1}$ in $\mathbb{S}^{3} \backslash L$. We denote the corresponding element in $\pi L$ by $k$. $k$ naturally inherits its orientation from $K$. Then $k$ is uniquely determined up to conjugation; i.e., the presentation of $k$ depends on the base point in $\mathbb{S}^{3} \backslash L$. Next, let $\psi$ be a representation of $\pi L$. 
Using $\pi L$ and $\psi$, we construct a $\mathcal{K} \psi(\pi L)$ module $V_{\psi}$, where $\mathcal{K}$ is a rational polynomial field. When the twisted Alexander polynomial of $L$ (see [5], [8]) does not vanish, we show that $V_{\psi}$ is generated by one element (Theorem 1 ). Therefore, if we fix a generator, as a coefficient of the generator, any $v$ in $V_{\psi}$ can be expressed by an element of $\mathcal{K} \psi(\pi L)$. Here we apply the "derivation" to $k$, whose consequence is an element of $V_{\psi}$. Hence it is also expressed by a matrix over $\mathcal{K}$. After some calculation, we can show that it is explicitly represented by a more simpler matrix determined by $k$ and the choice of the generator. We define $l k_{\psi}(K, L)$ in $\mathcal{K}$ by its determinant. It is remarkable that the definition of $l k_{\psi}$ itself does not need the condition that the twisted Alexander polynomial does not vanish. It can be shown that $l k_{\psi}(K, L)$ coincides with $l k(K, L)$ if $\psi$ is trivial. However, when $\psi$ is nontrivial, these two quantities do not necessarily coincide. We give such an example here.

\section{Preliminaries.}

2.1. Groups and Presentations. In this paper, we use the term 'groups' strictly in reference to finitely generated and finitely presented groups.

Let us begin by giving a brief summary of the points of combinatorial group theory [6] relevant to the present study. Let $\mathfrak{F}_{s}$ be a free group generated by $x_{1}, \ldots, x_{s}$, and let $\mathfrak{R}$ be a normal subgroup of $\mathfrak{F}_{s}$ generated by $r_{1}, \ldots, r_{t} \in \mathfrak{F}_{s}$. The elements of $\mathfrak{R}$ are of the form $\prod_{k=1}^{l} u_{k} r_{i_{k}}^{\varepsilon_{k}} u_{k}^{-1}$, where $u_{k} \in \mathfrak{F}_{s}$ and $\varepsilon_{k}= \pm 1$, and referred to as the consequences of $r_{1}, \ldots, r_{t}$. $\mathfrak{R}$ itself is also referred to as the consequence of $r_{1}, \ldots, r_{t} \cdot \mathfrak{F}_{s}$ and $\mathfrak{R}$ uniquely determine the quotient group $\mathfrak{G}=\mathfrak{F}_{s} / \mathfrak{R}$. Then, let $\phi$ be the associated homomorphism from $\mathfrak{F}_{s}$ to $\mathfrak{G}$ :

$$
\phi: \mathfrak{F}_{s}=\left\langle x_{1}, \ldots, x_{s}\right\rangle \rightarrow \mathfrak{G}=\left\langle x_{1}, \ldots, x_{s} \mid r_{1}, \ldots, r_{t}\right\rangle .
$$

We call $\left\langle x_{1}, \ldots, x_{s} \mid r_{1}, \ldots, r_{t}\right\rangle$ a presentation of $\mathfrak{G}$. The set $r_{1}, \ldots, r_{t}$ is called a set of relators, and the equations $r_{i}=1$ are called defining relations for $\mathfrak{G}$.

2.2. Tietze Transformations. A group $\mathfrak{G}$ can have many presentations. Tietze showed that if $\mathfrak{G}$ has two presentations, one can be transformed into the other by a finite sequence of Tietze transformations [6]. More precisely, he showed that if we are given a presentation

$$
\mathfrak{G}=\left\langle x_{1}, \ldots, x_{s} \mid r_{1}, \ldots, r_{t}\right\rangle,
$$

then any other presentation for $\mathfrak{G}$ can be obtained by using a finite number of the following transformations successively:

$\mathrm{T}_{1}$ : If a word $r$ is $r_{1}, \ldots, r_{t}$, then $r$ is added to the defining relators in $\mathfrak{G}$. The resulting presentation is

$$
\left\langle x_{1}, \ldots, x_{s} \mid r_{1}, \ldots, r_{t}, r\right\rangle .
$$


$\mathrm{T}_{2}$ : If $y$ is any word in $x_{1}, \ldots, x_{s}$, then $x$ is added to the generating symbols in $\mathfrak{G}$ and the word $x y^{-1}$ is added to the relators in $\mathfrak{G}$. The resulting presentation is

$$
\left\langle x_{1}, \ldots, x_{s}, x \mid r_{1}, \ldots, r_{t}, x y^{-1}\right\rangle \text {. }
$$

The transformations $\mathrm{T}_{1}, \mathrm{~T}_{1}{ }^{-1}, \mathrm{~T}_{2}$ and $\mathrm{T}_{2}{ }^{-1}$ are called Tietze transformations, where $T_{1}^{-1}$ and $T_{2}^{-1}$ are the inverse operations of $T_{1}$ and $T_{2}$, respectively.

2.3. Free Differential Calculus. We now review the theory of free differential calculus [3], [4].

Let $\mathfrak{G}$ be a group and let $\mathbb{Z} \mathfrak{G}$ be the group ring of $\mathfrak{G}$ over the integers. A derivation $D$ in $\mathbb{Z} \mathfrak{G}$ is defined as a mapping of $\mathbb{Z} \mathfrak{G}$ into itself with the properties

$$
\begin{aligned}
D(u+v) & =D u+u D v, \quad u, v \in \mathbb{Z} \mathfrak{G}, \\
D(u \cdot v) & =D u \cdot v^{\varepsilon}+u \cdot D v,
\end{aligned}
$$

where $v^{\varepsilon}$ is the sum of the coefficients of $v$. This definition implies that

$$
\begin{aligned}
D(a) & =0, & & a \in \mathbb{Z}, \\
D\left(g^{-1}\right) & =-g^{-1} D g, & & g \in \mathfrak{G}, \\
D\left(g^{n}\right) & =\left(1+g+g^{2}+\cdots+g^{n-1}\right) D g . & &
\end{aligned}
$$

The derivations in $\mathbb{Z} \mathfrak{G}$ form a right $\mathbb{Z} \mathfrak{G}$ module, where addition and right multiplication by $v$ are defined by

$$
\begin{array}{rlrl}
\left(D_{1}+D_{2}\right) u & =D_{1} u+D_{2} u, & u \in \mathbb{Z} \mathfrak{G}, \\
(D \cdot v) u & =D u \cdot v .
\end{array}
$$

In particular, when $\mathfrak{G}$ is the free group $\mathfrak{F}_{s}$, all derivations are explicitly determined in the following sense. For each generator $x_{j}$ of $\mathfrak{F}_{s}$, derivations $D_{x_{j}}=\partial / \partial x_{j}$ are partial derivations if they satisfy

$$
\frac{\partial x_{i}}{\partial x_{j}}=\delta_{i j}
$$

Fox showed that the partial derivations form a basis of the module of derivations [3]. That is, every derivation $D: \mathbb{Z} \mathfrak{F}_{s} \rightarrow \mathbb{Z} \mathfrak{F}_{s}$ is given by

$$
D=\sum_{j=1}^{s} \frac{\partial}{\partial x_{j}} D x_{j},
$$

where the $D x_{j}$ are the prescribed elements in $\mathbb{Z F}_{s}$. One can easily check that the map $D_{\varepsilon}(w)=w-w^{\varepsilon}$ is also a derivation. Hence, for any element $w \in \mathfrak{F}_{s}$, we have the following formula:

$$
w-1=\sum_{j=1}^{s} \frac{\partial w}{\partial x_{j}}\left(x_{j}-1\right) .
$$




\section{Alexander modules associated with representations.}

Let $\mathfrak{G}$ be a group, and let $\phi$ be the associated homomorphism presented in (1). We extend $\phi$ to a linear map on the group ring. (For simplicity we also denote this extension by $\phi$.) Let $J$ be the $t \times s$ matrix over $\mathbb{Z} \mathfrak{G}$ defined by

$$
J=\left(\phi\left(\frac{\partial r_{i}}{\partial x_{j}}\right)\right)=\left(\begin{array}{ccc}
\phi\left(\frac{\partial r_{1}}{\partial x_{1}}\right) & \ldots & \phi\left(\frac{\partial r_{1}}{\partial x_{s}}\right) \\
\vdots & & \vdots \\
\phi\left(\frac{\partial r_{t}}{\partial x_{1}}\right) & \ldots & \phi\left(\frac{\partial r_{t}}{\partial x_{s}}\right)
\end{array}\right) .
$$

$J$ is called the Jacobian.

Now, let $V$ be a $\mathbb{Z} \mathfrak{G}$ module whose generators are $d x_{1}, \ldots, d x_{s}$ and defining relations are given by $J$ :

$$
V=\left\langle d x_{1}, \ldots, d x_{s} \mid \sum_{j=1}^{s} \lambda\left(\phi\left(\frac{\partial r_{i}}{\partial x_{j}}\right)\right) d x_{j}=0(i=1, \ldots, t)\right\rangle,
$$

where $\lambda$ denotes an action on $V . V$ is called the Alexander module.

Next, let $\mathfrak{H}$ be a group and let $\psi$ be the homomorphism from $\mathfrak{G}$ to $\mathfrak{H}$. We extend $\psi$ to a $\mathbb{Z}$ linear map (and denote this extension by $\psi$ ). Let $V_{\psi}$ be the $\mathbb{Z} \mathfrak{H}$ module defined by

$$
V_{\psi}=\left\langle d x_{1}, \ldots, d x_{s} \mid \sum_{j=1}^{s} \lambda_{\psi}\left(\psi \phi\left(\frac{\partial r_{i}}{\partial x_{j}}\right)\right) d x_{j}=0(i=1, \ldots, t)\right\rangle,
$$

where $\lambda_{\psi}$ denotes an action on $V_{\psi}$. We call $V_{\psi}$ the Alexander module associated with the representation $\psi$. Note that $\psi$ naturally induces a linear map $\widetilde{\psi}$ from $V$ to $V_{\psi}$ by

$$
\widetilde{\psi}\left(\lambda(g) d x_{i}\right)=\lambda_{\psi}(\psi(g)) d x_{i} .
$$

Therefore we have the following commutative diagram:

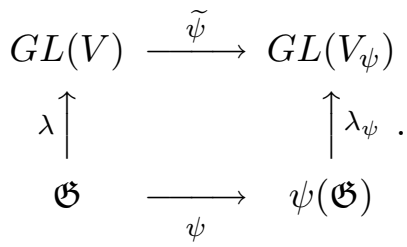

When there is no danger of confusion, we omit $\lambda$ and $\lambda_{\psi}$.

Formally, each relation in $V$ is derived as follows. Let $d$ be a derivation in $\mathbb{Z} \mathfrak{F}_{s}$. For an element $w$ in $\mathfrak{F}_{s}$, its derivation by $d$ is presented by $d(w)=$ $\sum_{j=1}^{s} \frac{\partial w}{\partial x_{j}} d x_{j}$. Here we define an element of $V$ by regarding $d x_{1}, \ldots, d x_{s}$ as generators and considering the $\phi$ images of each coefficient of $d(w)$. For convenience, we denote such an element by $\phi d(w)$. Then, each relation is defined by the relation $\phi d\left(r_{i}\right)=0$. 
Indeed, $V$ is a homology of the universal covering of the space $X$ satisfying $\pi_{1} X=\mathfrak{G}$. The covering transformation is $\pi_{1} X$ itself. For a closed path $x$ in $X$ whose corresponding element in $\pi_{1} X$ is $\phi(w), \phi d(w)$ is a lift of $x$. For more details, see [2], [7].

Let $\mathcal{K}$ be a commutative field such that there exits a natural inclusion or projection from $\mathbb{Z}$ to $\mathcal{K}$. We extend $V$ to a $\mathcal{K} \mathfrak{G}$ module by the map $\mathbb{Z} \rightarrow \mathcal{K}$. Similarly, we extend $V_{\psi}$ to a $\mathcal{K} \mathfrak{H}$ module, and $\mathbb{Z}$ linear maps $\phi$ and $\psi$ to $\mathcal{K}$ linear maps. Below, we consider a rational polynomial field $\mathbb{K}\left(t_{1}^{ \pm 1}, \ldots, t_{r}^{ \pm 1}\right)$ for $\mathcal{K}$, where $\mathbb{K}$ represents $\mathbb{C}, \mathbb{R}$ or a finite field $\mathbb{F}$.

Since the Jacobian depends on the choice of the presentation of $\mathfrak{G}$, let us consider the operations to which the Jacobian matrices are subject as induced by the Tietze transformations [9]. These operations are of the following three general types (and any operation belonging to one of the following three types is an operation induced by a Tietze transformation):

$\mathrm{J}_{0}$ : Permutation of rows or columns.

$\mathrm{J}_{1}$ : Adjoinment to $J$ of a new row that is a left $\mathbb{Z} \mathfrak{G}$ combination of the rows of $J$.

$\mathrm{J}_{2}$ : Adjoinment to $J$ of a new row and a new column satisfying the condition that the entry in their intersection is $1=1 \cdot e \in \mathbb{Z} \mathfrak{G}$ ( $e$ is the identity element of $\mathfrak{G}$ ) and the remaining entries in the new column are all zero:

$$
J \rightarrow\left(\begin{array}{cc}
J & 0 \\
* & 1
\end{array}\right)
$$

We consider two matrices over $\mathbb{Z} \mathfrak{G}$ to be equivalent if one can be obtained from the other by a finite sequence of operations of the types $J_{0}, J_{1}, J_{2}, J_{1}{ }^{-1}$ and $J_{2}{ }^{-1}$, where $J_{1}^{-1}$ and $J_{2}{ }^{-1}$ are the inverse operations of types $J_{1}$ and $\mathrm{J}_{2}$, respectively. It is well-known that the Jacobians of $\mathfrak{G}$ are all equivalent.

Since we would like to consider $\mathfrak{H}$ to be $G L\left(\mathcal{K}^{n}\right)$ and $\psi$ to be a representation of $\mathfrak{G}$ to $G L\left(\mathcal{K}^{n}\right)$, we define equivalence classes of matrices over $\Lambda=\operatorname{Mat}\left(\mathcal{K}^{n}\right)$ in terms of operations for the following types. (We use " $A$ " to represent an arbitrary such matrix.):

$\mathrm{t}_{0}$ : Permutation of the $i$-th block and the $j$-th block rows (or columns).

$\mathrm{t}_{1}$ : Adjoinment to $A$ of a new row that is a $\Lambda$ combination of the rows of $A$.

$\mathrm{t}_{2}$ : Adjoinment to $A$ of a new row and a new column satisfying the conditions that the entry in their intersection $1=1 \cdot E_{n}\left(E_{n}\right.$ is the identity matrix) and the remaining entries in the new column are all 0 :

$$
A \rightarrow\left(\begin{array}{cc}
A & 0 \\
* & 1
\end{array}\right)
$$

$\mathrm{t}_{3}$ : Addition, to the $i$-th row of $A$, of a $\Lambda$ combination of the rows in the complementary set. 
$t_{4}$ : Change of the basis for the representation.

Similarly to the case of the Jacobians, we consider two matrices over $\Lambda, A$ and $B$, to be equivalent if one can be obtained from the other by a finite sequence of operations of types $t_{0}, \ldots, t_{4}$, and $t_{1}{ }^{-1}, t_{2}{ }^{-1}, t_{3}{ }^{-1}$, where $t_{1}{ }^{-1}, t_{2}{ }^{-1}$ and $t_{3}{ }^{-1}$ are the inverse operations of types $t_{1}, t_{2}$ and $t_{3}$, respectively. We denote that matrices $A$ and $B$ are equivalent by $A \sim B$. When we would like to emphasize that $A$ is transformed to $B$ by $\mathrm{t}_{*}$, we write $A \stackrel{\mathrm{t}_{*}}{\sim} B$.

It is clear that if two groups $\mathfrak{G}_{1}$ and $\mathfrak{G}_{2}$ are isomorphic, then the presentation matrices of $V_{1}$ and $V_{2}$ are equivalent. Furthermore, if $\psi_{1}$ and $\psi_{2}$ are equivalent representations, the presentation matrices of $V_{1 \psi_{1}}$ and $V_{2 \psi_{2}}$ are also equivalent.

\section{Main results.}

In order to establish our main results, we need some preliminary discussion.

Lemma 1. Let $A$ be a $2 \times 2$ matrix whose entries are in $\Lambda$. Then, $A$ is nonsingular as a $2 n \times 2 n$ matrix if and only if

$$
A=\left(\begin{array}{cc}
A_{11} & A_{12} \\
A_{21} & A_{22}
\end{array}\right) \stackrel{\mathrm{t}_{0}, \mathrm{t}_{3}}{\sim}\left(\begin{array}{cc}
D_{1} & 0 \\
0 & D_{2}
\end{array}\right)
$$

where $D_{1}$ and $D_{2}$ are in $G L\left(\mathcal{K}^{n}\right)$.

Proof. Demonstrating the 'if' part is trivial, and we therefore present only the proof for the 'only if' part. If an entry in $A$ is a nonsingular matrix, we may assume that $A_{11}$ is nonsingular by an operation of type $t_{0}$. It is easy to see that

$$
\left(\begin{array}{cc}
E_{n} & 0 \\
-A_{21} A_{11}^{-1} & E_{n}
\end{array}\right)\left(\begin{array}{cc}
A_{11} & A_{12} \\
A_{21} & A_{22}
\end{array}\right)=\left(\begin{array}{cc}
A_{11} & A_{12} \\
0 & -A_{21} A_{11}^{-1} A_{12}+A_{22}
\end{array}\right) .
$$

Since $A$ is nonsingular, $-A_{21} A_{11}^{-1} A_{12}+A_{22}$ is also nonsingular. Then, considering $t_{3}$, we observe

$$
A \sim\left(\begin{array}{cc}
A_{11} & 0 \\
0 & -A_{21} A_{11}^{-1} A_{12}+A_{22}
\end{array}\right) .
$$

If all entries in $A$ are singular, then there exist $P$ in $G L\left(\mathcal{K}^{n}\right)$ and $Q$ in $G L\left(\mathbb{K}^{n}\right)$ such that

$$
P Q^{-1} A_{11} Q=\left(\begin{array}{cccc}
E_{k} & & & \\
& 0 & & \\
& & \ddots & \\
& & & 0
\end{array}\right),
$$

where $k=\operatorname{rank} A_{11}$. Note that we can choose matrices $P$ and $Q$ in such a way that $P$ consists of multiplications of matrices which induce an operation 
of type $t_{3}$ and $Q$ is a permutation matrix. For simplicity, we denote the matrix

$$
\left(\begin{array}{cc}
Q^{-1} & 0 \\
0 & E_{n}
\end{array}\right) A\left(\begin{array}{cc}
Q & 0 \\
0 & E_{n}
\end{array}\right)
$$

by $A_{Q}$. Then, for an appropriate matrix $P_{1}$, we have

$$
\begin{aligned}
A^{\prime} & =\left(\begin{array}{cc}
E_{n} & 0 \\
P_{1} & E_{n}
\end{array}\right)\left(\begin{array}{cc}
P & 0 \\
0 & E_{n}
\end{array}\right) A_{Q} \\
& =\left(\begin{array}{cc}
E_{n} & 0 \\
P_{1} & E_{n}
\end{array}\right)\left(\begin{array}{cc|c}
E_{k} & \vdots & P Q^{-1} A_{12} \\
\ldots & 0 & \\
\hline A_{21} Q & A_{22}
\end{array}\right) \\
& \stackrel{\underline{\mathrm{t}}_{3}}{=}\left(\begin{array}{cc|c}
E_{k} & \vdots & A_{12}^{\prime} \\
\ldots & 0 & \\
\hline 0 & A_{21}^{\prime} & A_{22}^{\prime}
\end{array}\right) .
\end{aligned}
$$

By the assumption, the $n \times(n-i)$ matrix $A_{21}^{\prime}$ must be of full rank. Next, we apply $t_{3}$ twice to each row of $A^{\prime}$. That is, for appropriate matrices $P_{2}$ and $P_{3}$, we have

$$
\begin{aligned}
& A^{\prime \prime}=\left(\begin{array}{cc}
E_{n} & 0 \\
P_{3} & E_{n}
\end{array}\right)\left(\begin{array}{cc}
E_{n} & P_{2} \\
0 & E_{n}
\end{array}\right) A^{\prime} \\
& \stackrel{\underline{\mathrm{t}_{3}}}{=}\left(\begin{array}{cc}
E_{n} & 0 \\
P_{3} & E_{n}
\end{array}\right)\left(\begin{array}{cc|c}
E_{k} & & A_{12}^{\prime \prime} \\
& E_{n-k} & \\
\hline 0 & A_{21}^{\prime} & A_{22}^{\prime}
\end{array}\right) \\
& =\left(\begin{array}{cc}
E_{n} & 0 \\
P_{3} & E_{n}
\end{array}\right)\left(\begin{array}{cc}
E_{n} & A_{12}^{\prime \prime} \\
A_{21}^{\prime \prime} & A_{22}^{\prime}
\end{array}\right) \\
& \stackrel{\mathrm{t}_{3}}{=}\left(\begin{array}{cc}
E_{n} & A_{12}^{\prime \prime} \\
0 & A_{22}^{\prime \prime}
\end{array}\right) \text {. }
\end{aligned}
$$


Also by the assumption, $A^{\prime \prime}$ and $A_{22}^{\prime \prime}$ must be nonsingular. Now, we summarize the above equations:

$$
\begin{aligned}
A^{\prime \prime} & =\left(\begin{array}{cc}
E_{n} & 0 \\
P_{3} & E_{n}
\end{array}\right)\left(\begin{array}{cc}
E_{n} & P_{2} \\
0 & E_{n}
\end{array}\right)\left(\begin{array}{cc}
E_{n} & 0 \\
P_{1} & E_{n}
\end{array}\right)\left(\begin{array}{cc}
P & 0 \\
0 & E_{n}
\end{array}\right) A_{Q} \\
& =\left(\begin{array}{cc}
P & 0 \\
0 & E_{n}
\end{array}\right)\left(\begin{array}{cc}
E_{n} & 0 \\
P_{3} P & E_{n}
\end{array}\right)\left(\begin{array}{cc}
E_{n} & P^{-1} P_{2} \\
0 & E_{n}
\end{array}\right)\left(\begin{array}{cc}
E_{n} & 0 \\
P_{1} P & E_{n}
\end{array}\right) A_{Q}, \\
\therefore & \left(\begin{array}{cc}
E_{n} & 0 \\
P_{3} P & E_{n}
\end{array}\right)\left(\begin{array}{cc}
E_{n} & P^{-1} P_{2} \\
0 & E_{n}
\end{array}\right)\left(\begin{array}{cc}
E_{n} & 0 \\
P_{1} P & E_{n}
\end{array}\right) A_{Q} \\
& =\left(\begin{array}{cc}
P^{-1} & 0 \\
0 & E_{n}
\end{array}\right) A^{\prime \prime} \\
& =\left(\begin{array}{cc}
P^{-1} & P^{-1} A_{12}^{\prime \prime} \\
0 & A_{22}^{\prime \prime}
\end{array}\right) .
\end{aligned}
$$

Therefore, by considering $t_{3}$, we have

$$
A_{Q} \stackrel{\mathrm{t}_{3}}{\sim}\left(\begin{array}{cc}
P^{-1} & 0 \\
0 & A_{22}^{\prime \prime}
\end{array}\right) .
$$

Finally, we obtain

$$
A \stackrel{\mathrm{t}_{3}}{\sim}\left(\begin{array}{cc}
Q P^{-1} Q^{-1} & 0 \\
0 & A_{22}^{\prime \prime}
\end{array}\right) .
$$

This completes the proof of the lemma.

We extend the statement of Lemma 1 from $2 \times 2$ matrices to $m \times m$ matrices.

Proposition 1. Let $A$ be an $m \times m$ matrix whose entries are in $\Lambda$. Then, $A$ is nonsingular as an $m n \times m n$ matrix if and only if

$$
A \stackrel{\mathrm{t}_{0}, \mathrm{t}_{3}}{\sim}\left(\begin{array}{ccc}
D_{1} & & 0 \\
& \ddots & \\
0 & & D_{m}
\end{array}\right),
$$

where $D_{1}, \ldots, D_{m}$ are in $G L\left(\mathcal{K}^{n}\right)$.

Proof. This proof is similar to that of Lemma 1. Suppose that operations of the types applied there succeed through the $(i-1)$-th row and fail at the $i$-th row:

$$
A \stackrel{\mathrm{t}_{0}, \mathrm{t}_{3}}{\sim}\left(\begin{array}{cccc}
A_{11} & & 0 & * \\
& \ddots & & \vdots \\
0 & & A_{i-1 i-1} & * \\
0 & \ldots & 0 & B
\end{array}\right), \quad B=\left(\begin{array}{ccc}
A_{i i} & \ldots & A_{i m} \\
\vdots & & \vdots \\
A_{m i} & \ldots & A_{m m}
\end{array}\right),
$$

where $A_{11}, \ldots, A_{i-1 i-1}$ are nonsingular, and each entry in $B$ is singular. Then it is enough into show that $A_{i i}$ can be transformed into a nonsingular 
matrix by an operation of type $t_{3}$. First, set $k=\operatorname{rank}\left(A_{i i}\right)<n$. Then, from the nonsingularity of $A$, we can increase the rank of $A_{i i}$ by at least one by using some $A_{j i}(j=i+1, \ldots, n)$, as in the proof of Lemma 1. Repeating this process at most $n-k$ times, we have a nonsingular matrix in the $(i, i)$-th entry of $A$. This concludes the proof.

Here, as $\mathfrak{G}$ we take the fundamental group of the complement of the link $L$ with $r$ components in $\mathbb{S}^{3}$ :

$$
\mathfrak{G}=\pi L=\left\langle x_{1}, \ldots, x_{s} \mid r_{1}, \ldots, r_{s-1}\right\rangle .
$$

Then, let $\rho$ be an irreducible representation of $\pi L$ :

$$
\rho: \pi L \rightarrow G L\left(\mathbb{K}^{n}\right) .
$$

Note that $\mathbb{K} \rho(\pi L)$ is isomorphic to the full matrix algebra $\operatorname{Mat}\left(\mathbb{K}^{n}\right)$ if and only if $\rho$ is irreducible. Because $\pi L$ is described by the Wirtinger presentation, there exits a natural abelianization homomorphism $\alpha$ from $\pi L$ to $\pi L / \pi L^{\prime}$ :

$$
\alpha: \pi L \rightarrow \pi L / \pi L^{\prime} \cong\left\langle t_{1}, \ldots, t_{r} \mid t_{i} t_{j}=t_{j} t_{i}(i, j=1, \ldots, r)\right\rangle .
$$

Next, let $\theta$ be a linear map from $\mathbb{K} \pi L$ to $\mathcal{K}=\mathbb{K}\left(t_{1}^{ \pm 1}, \ldots, t_{r}^{ \pm 1}\right)$ that factors through $\alpha$ :

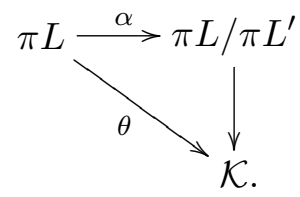

We use the same symbol $t_{i}$ in $\pi L / \pi L^{\prime}$ and $\mathcal{K}$ for convenience. Let $\psi$ be a representation of $\pi L$,

$$
\psi: \pi L \rightarrow G L\left(\mathcal{K}^{n}\right),
$$

defined by $\psi\left(x_{i}\right)=\theta\left(x_{i}\right) \rho\left(x_{i}\right)$. Then the irreducibility of $\rho$ implies

$$
\mathcal{K} \psi(\pi L)=\operatorname{Mat}\left(\mathcal{K}^{n}\right) .
$$

Therefore we can apply Proposition 1 to the presentation matrix of $V_{\psi}$.

Let us recall the twisted Alexander polynomial [5], [8]. Then, let $R_{\hat{k}}$ be an $(s-1) \times(s-1)$ submatrix of presentation matrix of $V$ obtained by removing the $k$-th column. Let $\psi\left(R_{\hat{k}}\right)$ be an $(s-1) \times(s-1)$ matrix whose $(i, j)$-th entry is the $\psi$ image of the $(i, j)$-th entry of $R_{\hat{k}}$. Hence, as a matrix, we can see that $\psi\left(R_{\hat{k}}\right)$ is an $n(s-1) \times n(s-1)$ matrix. Then, the twisted Alexander polynomial $\Delta_{\pi L, \psi}=\Delta_{\pi L, \psi}\left(t_{1}, \ldots, t_{r}\right)$ is defined by

$$
\Delta_{\pi L, \psi}=\frac{\operatorname{det} \psi\left(R_{\hat{k}}\right)}{\operatorname{det} \psi\left(1-x_{k}\right)} \text {. }
$$

In our setting, the denominator of $\Delta_{\pi L, \psi}$ is essentially the characteristic polynomial of the nonsingular matrix $\rho\left(x_{k}\right)$ because we use the Wirtinger 
presentation. This definition is independent of the choice of the index $k$ and the presentation of $\pi L$ up to a factor $\pm t_{1}^{e_{1}} \ldots t_{r}^{e_{r}}$, where $e_{1}, \ldots, e_{r}$ are integers.

Proposition 2. $\Delta_{\pi L, \psi}$ does not vanish if and only if

$$
\psi\left(R_{\hat{k}}\right) \stackrel{\mathrm{t}_{0}, \mathrm{t}_{3}}{\sim}\left(\begin{array}{ccc}
D_{1} & & 0 \\
& D_{2} & \\
0 & & \ddots
\end{array}\right),
$$

where $D_{1}, D_{2}, \ldots$ are nonsingular matrices.

Proof. $\Delta_{\pi L, \psi} \neq 0$ if and only if $\operatorname{det} \psi\left(R_{\hat{k}}\right) \neq 0$ for any $k$, which implies that $\psi\left(R_{\hat{k}}\right)$ is nonsingular. The conclusion follows from Proposition 1.

In the remainder of this paper, we assume that $\Delta_{\pi L, \psi}$ does not vanish. By Proposition 2, we have

$$
\left(\psi \phi\left(\frac{\partial r_{i}}{\partial x_{j}}\right)\right) \stackrel{\mathrm{t}_{0}, \mathrm{t}_{3}}{\sim}\left(\begin{array}{cccc}
A_{1} & & & B_{1} \\
& \ddots & & \\
& & A_{s-1} & B_{s-1}
\end{array}\right),
$$

where $B_{i} \in \operatorname{Mat}\left(\mathcal{K}^{n}\right)(i=1, \ldots, s-1)$. Because (3) represents a transformation consisting of operations of types $t_{0}$ and $t_{3}$, we obtain

$$
V_{\psi} \cong\left\langle d x_{1}, \ldots, d x_{s} \mid A_{i} d x_{i}+B_{i} d x_{s}=0(i=1, \ldots, s-1)\right\rangle .
$$

Next, we determine each $B_{i}$. From (2), we have

$$
\psi \phi(w)-1=\sum_{j=1}^{s} \psi \phi\left(\frac{\partial w}{\partial x_{j}}\right)\left(\psi \phi\left(x_{j}\right)-1\right) .
$$

If we choose the relator $r_{i}$ as $w$, then

$$
\sum_{j=1}^{s} \psi \phi\left(\frac{\partial r_{i}}{\partial x_{j}}\right)\left(\psi \phi\left(x_{j}\right)-1\right)=0 .
$$

These equations show that the presentation matrix has the property that the sum of each row multiplied by $\psi \phi\left(x_{j}\right)-1$ vanishes. Furthermore, it is obvious that operations of types $t_{0}$ and $t_{3}$ do not change this property. Hence, we obtain

$$
A_{i}\left(\psi \phi\left(x_{i}\right)-1\right)+B_{i}\left(\psi \phi\left(x_{s}\right)-1\right)=0 .
$$

Since we use the Wirtinger presentation as a presentation of $\pi L$, the matrix $\psi \phi\left(x_{s}\right)$ does not have an eigenvalue 1 . This implies that $\psi \phi\left(x_{s}\right)-1$ is invertible, and we have

$$
B_{i}=-A_{i}\left(\psi \phi\left(x_{i}\right)-1\right)\left(\psi \phi\left(x_{s}\right)-1\right)^{-1} .
$$


Then, since each $A_{i}$ in $\left(\psi \phi\left(\frac{\partial r_{i}}{\partial x_{j}}\right)\right)$ is a nonsingular matrix, we obtain

$$
\begin{aligned}
V_{\psi} \cong\left\langle d x_{1}, \ldots, d x_{s}\right| d x_{i}-\left(\psi \phi\left(x_{i}\right)-1\right)\left(\psi \phi\left(x_{s}\right)-1\right)^{-1} d x_{s}=0 \\
(i=1, \ldots, s-1)\rangle .
\end{aligned}
$$

Therefore, for any element $v$ in $V_{\psi}$, we can write $v=v_{s} d x_{s}$, where $v_{s} \in$ $\operatorname{Mat}\left(\mathcal{K}^{n}\right)$. This means that $V_{\psi}$ is generated by $d x_{s}$.

Summarizing the above discussion, we have the following theorem.

Theorem 1. For any $i=1, \ldots, s, V_{\psi}$ is generated by $d x_{i}$ :

$$
V_{\psi}=\mathcal{K} \psi(\pi L) d x_{i} .
$$

Now, we fix the index of the generator of $V_{\psi}$, denoted by $s$. Let $c$ be an element of $\mathfrak{F}_{s}$ described by $c=x_{i_{1}} x_{i_{2}} x_{i_{3}} \ldots$ Then

$$
\begin{aligned}
& \widetilde{\psi} \phi(d(c))= d x_{i_{1}}+\psi \phi\left(x_{i_{1}}\right) d x_{i_{2}}+\psi \phi\left(x_{i_{1}} x_{i_{2}}\right) d\left(x_{i_{3}} \ldots\right) \\
&=\left(\psi \phi\left(x_{i_{1}}\right)-1\right)\left(\psi \phi\left(x_{s}\right)-1\right)^{-1} d x_{s} \\
& \quad \quad+\psi \phi\left(x_{i_{1}}\right)\left(\psi \phi\left(x_{i_{2}}\right)-1\right)\left(\psi \phi\left(x_{s}\right)-1\right)^{-1} d x_{s}+\ldots \\
&=\left(\psi \phi\left(x_{i_{1}}\right) \psi \phi\left(x_{i_{2}}\right)-1\right)\left(\psi \phi\left(x_{s}\right)-1\right)^{-1} d x_{s} \\
& \quad \quad+\psi \phi\left(x_{i_{1}}\right) \psi \phi\left(x_{i_{2}}\right) d\left(x_{i_{3}} \ldots\right) \\
&= \ldots \\
&=(\psi \phi(c)-1)\left(\psi \phi\left(x_{s}\right)-1\right)^{-1} d x_{s} .
\end{aligned}
$$

Similarly, for any element $c \in \mathfrak{F}_{s}$, we have

$$
\widetilde{\psi} \phi(d(c))=(\psi \phi(c)-1)\left(\psi \phi\left(x_{s}\right)-1\right)^{-1} d x_{s} .
$$

This calculation leads us to the following definitions.

Definition 1. Let $k$ be the element of $\pi L$ corresponding to $K$ for a suitable base point, and let $c$ be an element of $\mathfrak{F}_{s}$ satisfying $k=\phi(c)$. Then we define $L k_{\psi}(K, L)$ in $\operatorname{Mat}\left(\mathcal{K}^{n}\right)$ and $l k_{\psi}(K, L)$ in $\mathcal{K}$ by

$$
\begin{aligned}
L k_{\psi}(K, L) & =(\psi \phi(c)-1)\left(\psi \phi\left(x_{s}\right)-1\right)^{-1}, \\
l k_{\psi}(K, L) & =\operatorname{det}\left(L k_{\psi}(K, L)\right) .
\end{aligned}
$$

Remark 1. The condition such that $\Delta_{\pi L, \psi}$ does not vanish leads $V_{\psi}$ to be 1-dimensional, and furthermore if $c \in \mathfrak{F}_{s}, \widetilde{\psi} \phi(d(c))$ can be explicitly described by (4). Conversely if we accept the definition of $L k_{\psi}$ and $l k_{\psi}$ as Definition 1, we need not the condition.

Remark 2. If we choose another base point of $k$, or if we choose another index such that the component to which the index belongs is the same component to which $s$ belongs, then $L k_{\psi}$ changes to $P_{1}(\psi \phi(c)-1) P_{1}^{-1} P_{2}\left(\psi \phi\left(x_{s}\right)-\right.$ 1) $P_{2}^{-1}$, where $P_{1}, P_{2} \in G L\left(\mathbb{K}^{n}\right)$. If we take a representation of $\mathfrak{G}$ equivalent to $\rho$, then $L k_{\psi}$ also changes to $Q(\psi \phi(c)-1)\left(\psi \phi\left(x_{s}\right)-1\right) Q^{-1}$, where $Q \in G L\left(\mathbb{K}^{n}\right)$. We thus obtain the following theorem. 
Theorem 2. The definition of $l k_{\psi}$ does not depend on the choice of the base point. Furthermore, if we choose representations $\rho$ and $\theta$ satisfying all $\rho\left(x_{i}\right)$ to be conjugate with each other and all $\theta\left(x_{i}\right)$ to be the same monomial $t$, then $l k_{\psi}$ does not depend on the choice of the index $s$. Therefore, in this case, $l k_{\psi}$ only depends on the group $\mathfrak{G}$ and its representation $\rho($ or $\psi)$.

The condition stated in Theorem 2 obviously holds when $\rho$ is trivial and $t_{i}=t$ for $i=1, \ldots, r$.

Proposition 3. If $\rho$ is trivial and $t=t_{1}=\cdots=t_{r}$, then we have

$$
l k_{\psi}(K, L)=\frac{t^{n}-1}{t-1},
$$

where $n$ is the exponent of $k$.

Proof. If $\rho$ is trivial, we have $\psi \phi\left(x_{1}\right)=\cdots=\psi \phi\left(x_{s}\right)=t$. Let $c=$ $\prod_{j=1}^{m} x_{i_{j}}^{\varepsilon_{j}}\left(\varepsilon_{j}= \pm 1\right)$ be the element of $\mathfrak{F}_{s}$ satisfying $k=\phi(c)$. Then $\psi(k)=$ $\psi \phi(c)=t^{n}$, where $n=\sum_{j=1}^{m} e_{j}$. This concludes the proof.

Back to the definition of the linking number [7], we can easily see that $n$ appeared in the proof of Proposition 3 is equal to the linking number. Hence, in this case, we obtain the following corollary.

Corollary 1. Under the assumption of Proposition 3, we have

$$
l k_{\psi}(K, L) \stackrel{t \rightarrow 1}{\longrightarrow} \begin{cases}l k(K, L), & \text { if } \mathbb{K}=\mathbb{C} \text { or } \mathbb{R}, \\ l k(K, L) \bmod p, & \text { if } \mathbb{K}=\mathbb{F} p .\end{cases}
$$

Thus in this case $l k_{\psi}$ is equal to the linking number.

\section{Example.}

Let $W$ be Whitehead's link. Then $\pi W$ is generated by three generators $x$, $y$ and $z$, and its presentation is described by

$$
\pi W=\left\langle x, y, z \mid z y z^{-1} y z y^{-1} z^{-1} x^{-1}, x z x z^{-1} x^{-1} z y^{-1} z^{-1}\right\rangle .
$$

Let $K$ be an embedding of $\mathbb{S}^{1}$ in $\mathbb{S}^{3} \backslash W$ which is parallel to one component, as illustrated in Figure 1.

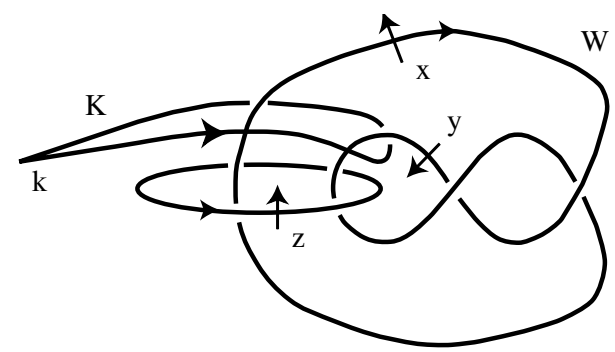

Figure 1. Whitehead's link. 
We consider a representation $\rho$ to $S L_{2}\left(\mathbb{F}_{5}\right)$ such that the image of each generator belongs to the conjugacy class of $\left(\begin{array}{ll}1 & 1 \\ 0 & 1\end{array}\right)$. This representation is described by

$$
\begin{gathered}
\rho: \pi W \rightarrow S L_{2}\left(\mathbb{F}_{5}\right) \\
\rho(x)=\left(\begin{array}{ll}
1 & 1 \\
0 & 1
\end{array}\right), \rho(y)=\left(\begin{array}{ll}
0 & 1 \\
4 & 2
\end{array}\right), \rho(z)=\left(\begin{array}{ll}
4 & 1 \\
1 & 3
\end{array}\right) .
\end{gathered}
$$

Such a representation onto $S L_{2}\left(\mathbb{F}_{5}\right)$ is unique up to inner automorphisms. We set $t=t_{1}=t_{2}$ and choose the index $x$ as a generator of $V_{\psi}$. Then,

$$
\begin{aligned}
L k_{\psi}(K, W) & =\left\{\psi\left(y^{-1} x\right)-\left(\begin{array}{ll}
1 & 0 \\
0 & 1
\end{array}\right)\right\}\left\{\psi(x)-\left(\begin{array}{ll}
1 & 0 \\
0 & 1
\end{array}\right)\right\}^{-1} \\
& =\left\{\left(\begin{array}{ll}
2 & 1 \\
1 & 1
\end{array}\right)-\left(\begin{array}{ll}
1 & 0 \\
0 & 1
\end{array}\right)\right\}\left\{t\left(\begin{array}{ll}
1 & 1 \\
0 & 1
\end{array}\right)-\left(\begin{array}{ll}
1 & 0 \\
0 & 1
\end{array}\right)\right\}^{-1} \\
& =\frac{1}{(t-1)^{2}}\left(\begin{array}{cc}
t-1 & -1 \\
t-1 & -t
\end{array}\right),
\end{aligned}
$$

and

$$
l k_{\psi}(K, W)=\frac{4}{(t-1)^{2}} .
$$

On the other hand, the linking number $l k(K, W)$ is 0 . This demonstrates that $K$ is a nontrivial embedding in $\mathbb{S}^{3} \backslash W$.

Acknowledgment. The author is grateful to Professor M. Wakayama for his continuous encouragement at Shikotsuko.

\section{References}

[1] J.S. Birman, Braids, links, and mapping class groups, Annals of Mathematics Studies, 82, Princeton University Press, Princeton, N.J.; University of Tokyo Press, Tokyo, 1974, MR 51 \#11477, Zbl 0305.57013.

[2] G. Burde and H. Zieschang, Knots, de Gruyter Studies in Mathematics, 5, Walter de Gruyter \& Co., Berlin-New York, 1985, MR 87b:57004, Zbl 0568.57001.

[3] R.H. Fox, Free differential calculus, I. Derivation in the free group ring, Ann. of Math. (2), 57 (1953), 547-560, MR 14,843d, Zbl 0050.25602.

[4] - Free differential calculus, II. The isomorphism problem of groups, Ann. of Math. (2), 59 (1954), 196-210, MR 15,931e, Zbl 0055.01704.

[5] B.J. Jiang and S.C. Wang, Twisted topological invariants associated with representations, Topics in knot theory (Erzurum, 1992), 211-227, NATO Adv. Sci. Inst. Ser. C Math. Phys. Sci., 399, Kluwer Acad. Publ., Dordrecht, 1993, CMP 1257 911, Zbl 0815.55001.

[6] W. Magnus, A. Karrass and D. Solitar, Combinatorial Group Theory, Second revised edition, Dover Publications, Inc., New York, 1976, MR 54 \#10423, Zbl 0362.20023. 
[7] D. Rolfsen, Knots and links, Mathematics Lecture Series, 7, Publish or Perish, Inc., Berkeley, Calif., 1976, MR 58 \#24236, Zbl 0339.55004.

[8] M. Wada, Twisted Alexander polynomial for finitely presentable groups, Topology, 33(2) (1994), 241-256, MR 95g:57021, Zbl 0822.57006.

[9] H.J. Zassenhaus, The Theory of Groups, 2nd ed. Chelsea Publishing Company, New York, (1958), x+265, MR 19,939d, Zbl 0083.24517.

Received September 27, 2000 and revised October 11, 2001. The author was partly supported by the Encouragement of Young Scientists, the Ministry of Education, Science, Culture and Sports, Japan (11740037).

Department of Mathematics

KANAZAWA UNIVERSITY

KAKUMA, KanaZAWA 920-1192

JAPAN

E-mail address: kawagoe@kappa.s.kanazawa-u.ac.jp 